\title{
Erratum: Orientations of low-energy domain walls in perovskites with oxygen octahedral tilts
} [Phys. Rev. B 90, 220101(R) (2014)]

Fei Xue, Yijia Gu, Linyun Liang, Yi Wang, and Long-Qing Chen (Received 27 January 2016; published 25 February 2016)

DOI: 10.1103/PhysRevB.93.059902

In the Acknowledgment of the original article, the Award No. DMR-0820404 should be replaced by DMR-1420620. 